\title{
SURFACE-BASED METHOD TO EVALUATE GLOBAL BRAIN SHAPE ASYMMETRIES IN HUMAN AND CHIMPANZEE BRAINS
}

\author{
Marc Fournier ${ }^{1}$, Benô̂t Combès ${ }^{1}$, Neil Roberts ${ }^{2}$, Simon Keller ${ }^{3}$, \\ Tim J. Crow ${ }^{4}$, William D. Hopkins ${ }^{5,6}$ and Sylvain Prima ${ }^{1}$ \\ ${ }^{1}$ INSERM, IRISA-INRIA VisAGeS Project-Team, F-35042 Rennes, France \\ ${ }^{2}$ CRIC, Queen's Medical Research Institute, Edinburgh, U.K. \\ ${ }^{3}$ Department of Neurology, University of Münster, 48149 Münster, Germany \\ ${ }^{4}$ SANE POWIC, Warneford Hospital, University of Oxford, UK \\ ${ }^{5}$ Department of Psychology, Agnes Scott College, Decatur, Georgia \\ ${ }^{6}$ Division of Psychobiology, Yerkes National Primate Research Center, Atlanta, Georgia
}

\begin{abstract}
In this paper we use humans and chimpanzees brain MRI databases to develop methods for evaluating global brain asymmetries. We perform brain segmentation and hemispheric surface extraction on both populations. The human brain segmentation pipeline is adapted to chimpanzees in order to obtain results of good quality. To alleviate the problems due to cortical variability we propose a mesh processing algorithm to compute the brain global shape. Surface-based global brain asymmetries are computed on chimpanzee and human subjects using individual mid-sagittal plane evaluation and populationlevel mean shape estimation. Asymmetry results are presented in terms of axis-wise components in order to perform more specific evaluation and comparison between the two populations.
\end{abstract}

Index Terms - Chimps MRI brain segmentation, Brain global shape computation, Surfaced-based bilateral brain asymmetries, Axis-wise asymmetry components, Chimp and human asymmetries comparison.

\section{INTRODUCTION}

Anatomical asymmetries of the human brain [1-3] have been known since the descriptions of asymmetries of the lateral Sylvian fissure by Eberstaller and Cunningham in the nineteenth century, but were largely forgotten until the rediscovery of the asymmetry of the planum temporale by Geschwind and Levitsky in 1968. Left larger than right planum temporale, as well as protruding right frontal and left occipital lobes (often termed the petalia or Yakovlevian torque), are the most striking asymmetrical features in most human brains. These asymmetries have been hypothesized to be related with brain functions, and are most often met in right-handed people whose language areas are mainly located on the left side of the brain.
These patterns of anatomical/functional asymmetries have long been thought to be typical of human beings (Homo sapiens) [4]. There has been much debate recently on this puzzling question [5], and some have claimed that these asymmetry patterns are actually shared by other great apes [6, 7]. Answering this key question could have important implications in the understanding of language origins and in what makes the humans unique.

Some techniques have been devised for the assessment of brain asymmetries in brain MR images of humans. Three approaches have been especially used. Most asymmetry studies are based on voxel-based morphometry (VBM) and variants [8-10]. Two other alternative techniques have also shown promising results, based on deformation-based morphometry (DBM) [11, 12] and low-dimensional analysis of asymmetries (LowD) [13]. However, to our knowledge, only voxel-based morphometry technique [14] and related volume-based analysis [15-18] have been applied to chimpanzees.

In this paper, we propose a new automated surface-based technique for the analysis of global shape asymmetries in humans and chimpanzees. First, the brain is segmented (and its hemispheres are separated) from high-resolution T1weighted 3T MR images, and its envelope is computed to assess its global shape. Second, asymmetries of this global brain shape are computed on an individual basis. A mean brain shape is then computed over a population of subjects, on which individual asymmetries are mapped for statistical analysis of the population asymmetries. Finally the output of these analyses on humans and chimpanzees are compared. Section 2 of this paper describes the material and methods: the segmentation pipeline, the brain global shape computation, the brain bilateral asymmetries evaluation, and the brain mean shape estimation. In Section 3 we present preliminary results on humans and chimpanzees obtained using the new technique, before concluding in Section 4. 


\section{MATERIAL AND METHODS}

This section describes the subject databases and the methods designed to process the datasets. We use two databases of MR images, the first one of humans and the second one of chimpanzees, composed of 30 subjects each. We focus our study on a subgroup of the population represented in the databases in order to limit the inter-subject variability. We choose to analyze and compare humans and chimpanzees asymmetries on the right-handed young adult male subjects of both populations. For the two populations, this group shows significant asymmetry results. For the chimpanzees this group is composed of 7 subjects from 12 to 26 years old and for the humans the group is composed of 11 healthy control subjects from 21 to 35 years old.

\subsection{Segmentation pipeline}

There are many softwares available to process MR images in order to obtain human brain segmentation. Most of these tools use a human brain atlas to register the data in the segmentation process. Methods relying on human brain atlases do not work for chimpanzees because their brain anatomy is quite different from the human one. Figure 1 shows some of these differences on MR image slices. To our knowledge no chimpanzee brain atlas is available yet to use standard atlas-based methods for brain segmentation.

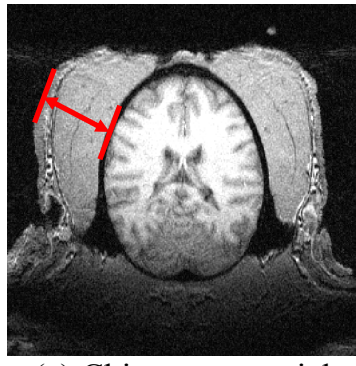

(a) Chimpanzee axial

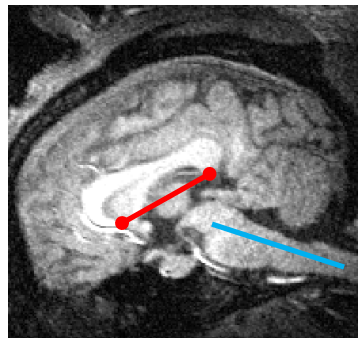

(c) Chimpanzee sagittal

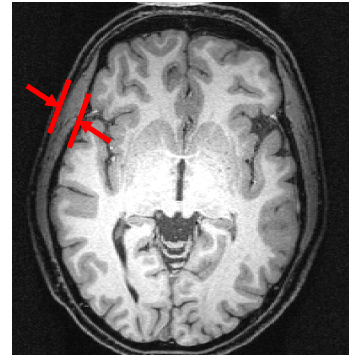

(b) Human axial

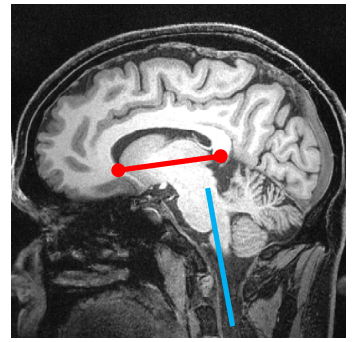

(d) Human sagittal
Fig. 1. Human and chimpanzee brain differences. (a) and (b) axial views show the surrounding tissues of the brain are thicker in chimpanzee. (c) and (d) sagittal views show different relative orientations of the anterior-posterior commissures and the spinal cord. (a) and (b) show on the same scale that the chimpanzee brain size is smaller than the human one.
While working with chimpanzee brain images, we need to define a pipeline without the use of an atlas. First we use BrainMask software (http://www.nitrc.org/projects/brainmask/) for the bias correction step followed by the initial brain mask evaluation for the whole brain. BrainMask provides an automatic and robust algorithm without atlas which works with chimpanzee brains. Then we use BrainVisa software (http://brainvisa.info/) to perform hemisphere split, surface extraction and brain alignment to the coordinate system axes based on anterior-posterior commissures landmarks. We use BrainVisa standard atlas-based process for human brains and its atlas-free feature for chimpanzee brains. In the atlasfree case for chimpanzees, we also use BrainVisa advanced correction and validation tools which provide an iterative threshold feature to obtain relevant results of hemisphere split.

\subsection{Brain global shape computation}

To alleviate the problems due to cortical variability, we base our asymmetry measures on a simplified yet accurate representation of the brain which closely follows the gyri without entering the sulci. We call this global shape the brain hull which we compute for each hemisphere obtained from BrainVisa output.

We first compute the 3D convex hull of each brain hemisphere vertices. This algorithm generates two meshes with few large triangles corresponding to coarse representations of each brain hemisphere which is not sufficiently accurate for our purpose. We subdivide these meshes to obtain a uniform triangulation with enough vertices to accurately describe the initial hemispheric brain meshes gyri positions. Then for each vertex of the subdivided convex hulls, we compute the minimal sphere centered on this vertex which intersects the boundary of the initial detailed hemispheric brain meshes. Finally we move the vertex in its opposite normal direction (towards the brain surface) on the minimal sphere surface.

This procedure illustrated in Figure 2(a) attracts vertices to the gyri positions and prevents them to penetrate inside the sulci, between neighboring gyri. It produces accurate hemispheric brain hulls shown in Figure 2(b) with the underlying hemispheric brain surfaces for a human subject.

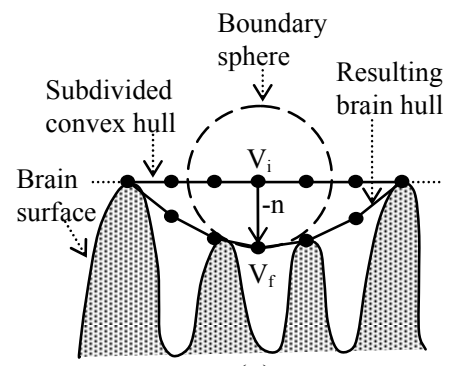

(a)

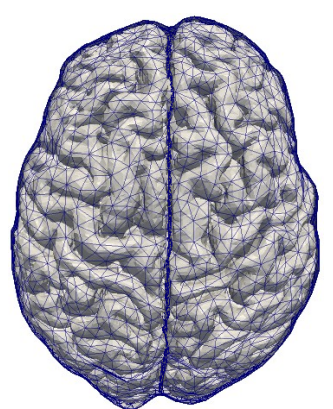

(b) 


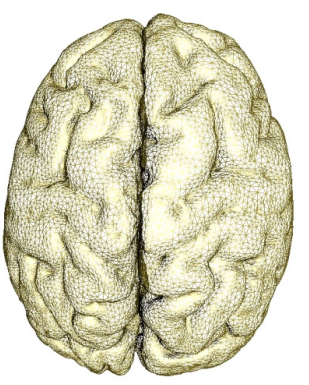

(c)

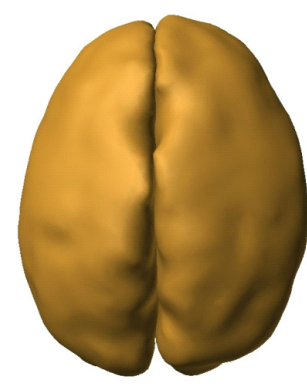

(d)
Fig. 2. Brain hull mesh processing illustration. (a) Brain hull algorithm computation with: $V_{i}$ the initial vertex position of the subdivided convex hull; $\mathrm{V}_{\mathrm{f}}$ the final vertex position of the brain hull; $-n$ the opposite normal vertex displacement direction. (b) Brain hull meshes superposed on a human brain hemispheric surfaces. (c) A chimpanzee brain hemispheric meshes output from BrainVisa. (d) The chimpanzee hemispheric brain hulls computed.

\subsection{Brain bilateral asymmetries evaluation}

The typical Yakovlevian torque human brain asymmetry of right-handed subjects is illustrated in Figure 3 as a combination of patterns which are exaggerated here to highlight this phenomenon. To quantify the magnitude of this torque we present a surface-based method to compute individual asymmetry maps (Section 2.3.1). These maps are defined over a vector deformation field that is decomposed in the three orthogonal axes. Then we explain how to put the individual asymmetry maps in a common coordinate system to perform statistical analyses (Section 2.3.2). The next two subsections provide specific implementation procedures on this strategy and additional methodological details can be found in [20-22].

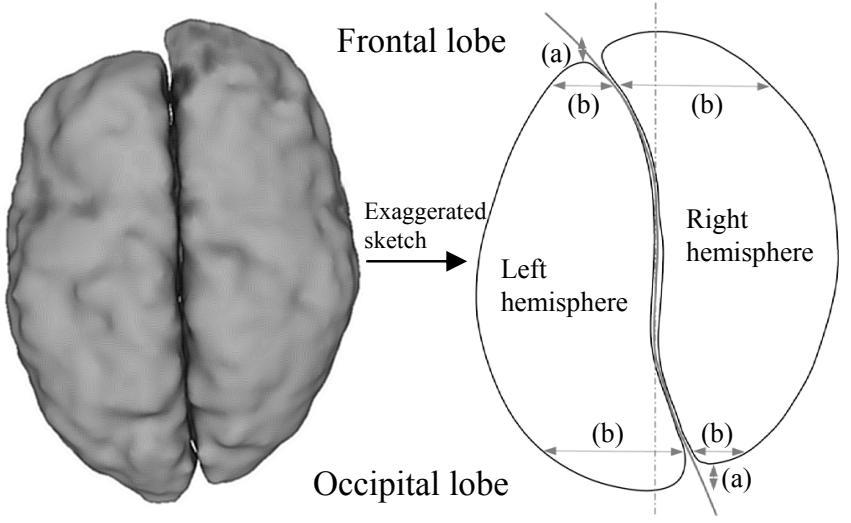

Fig. 3. Global brain asymmetries in right-handed human subjects when viewed from above. (a) Anterior (resp. posterior) protrusion of the brain in the right as compared to the left frontal lobe (resp. in the left as compared to the right occipital lobe). (b) Leftward frontal lobe and rightward occipital lobe deviation of the inter-hemispheric fissure.

\subsubsection{Generation of individual asymmetry maps}

We consider $n$ meshes $X_{1}, \ldots, X_{n}$ representing the $n$ brains under study, independently for each population (human and chimpanzee separated). For each of these $n$ meshes of a population, we propose to perform the following process.

- First step: Finding the symmetry plane.

We define the approximate symmetry plane $P$ of a mesh $X$ as the one best superposing $X$ and its reflection $S_{P}(X)$ about $P$. For that, we consider $X$ as a noised version of $S_{P}(X)$. This allows to consider each point $x i$ of $X$ as the realization of a random variable whose distribution is a mixture model composed of $\operatorname{card}(X)$ Gaussian laws $N\left(S_{P}\left(x_{k}\right), \sigma^{2} I\right), x_{k} \in X$. Then we define the optimal reflection $S_{P}$ as a ML estimate, which is computed using an EM algorithm. The use of a multiscale strategy and of a truncated Gaussian kernel allows a fast, accurate and robust estimation of the plane [20].

- Second step: Registering $X$ on $S_{P}(X)$.

Once $\mathrm{P}$ is known, we define the asymmetry field of $X$ as $(t(x)=T(x)-x)_{x \in X}$ where $T$ is the non linear transformation best superposing $X$ and $S_{P}(X)$. For that, we consider $T$ $\left(S_{P}(X)\right)$ as a noised version of $X$ (here $S_{P}$ is known). Then we design a MAP approach by specifying i) a prior on $T$ (a global affine model + a local order-one Tikhonov regularization on the linear component $\left.(\mathrm{t}(\mathrm{x}))_{-} \mathrm{x}\right)$, ii) a mixture density for the set $T\left(S_{P}(X)\right)$ and iii) a priori probabilities of matching between points of the two meshes (using geometrical descriptors almost invariant to the unknown transformation). Then, this MAP problem is solved using an EM algorithm [21].

\section{- Third step: Mapping the asymmetry field}

In order to provide specific results, we choose to analyze the asymmetry field by looking at its three components separately. This simply consists in projecting the asymmetry vector on the three coordinate axes and this yields the:

- postero-anterior component (difference in protrusion);

- left-right component (difference in width);

- head-foot component.

In the following, we call $s_{i}^{X}$ the scalar asymmetry mapping at point $x_{i}$ of mesh $X$ and $S^{X}$ the asymmetry map of $X$. Each subject has thus three asymmetry maps.

\subsubsection{Generation of a mean shape \& projection of the mapping}

- First step: Computing the mean shape

Individual asymmetry maps $S^{X}\left(X \in\left\{X_{1}, \ldots, X_{n}\right\}\right)$ have to be put in a common geometry to be compared. For this purpose, we compute the mean mesh $M$, defined as the mesh closest to all the meshes in the dataset (in a sense to be defined). In practice, we formulate the mean shape 
estimation as an iterative scheme that consists in the successive estimation of i) the mean shape and ii) the optimal similarity transformations between each mesh and the mean mesh [22].

\section{- Second step: Individual projection of the asymmetry mapping}

Together with the mean shape $M$, the previous algorithm provides us with the a posteriori probability that each point $m_{i}$ of $M$ is matched with a point $x_{j}$ of $X$; we call this probability $A_{i j}\left(\sum_{j} A_{i j}=1\right)$. This probability is used to project each individual asymmetry map on $M$. We call $s_{i}^{X \rightarrow M}$ the mapping information of $X$ projected on point $m_{i}$ of $M$ and define it as $s_{i}^{X \rightarrow M}=\sum_{j} A_{i j} s_{i}^{X}$.

\section{- Third step: Population statistical asymmetry maps}

At this point we now have a collection of $n(\times 3)$ scalar asymmetry maps $S^{X_{1} \rightarrow M}, \ldots ., S^{X_{n} \rightarrow M}$ projected on a common mean mesh $M$. We compute a point-wise mean and t-test (corrected for multiple comparisons) on each of the three components of the asymmetry field over the population. The correction for multiple comparisons is performed as described in [23] by i) fixing a supra-threshold $p=0.05$, ii) building the permutation distribution of the maximal suprathreshold cluster size (by shuffling $p$-values over the mesh and computing the size of the largest cluster of significant vertices, $n b T e s t=20.000$ permutations) iii) considering the critical supra-threshold cluster size as the $\left[\alpha^{*} n b T e s t\right]+1$ largest value over the sampling distribution and iv) removing from the statistical map the clusters having a size smaller than the critical supra-threshold cluster size.

\section{RESULTS AND DISCUSSION}

\subsection{Asymmetry maps interpretation}

For a correct interpretation of the directional asymmetry maps results computed and presented in this section, one needs to understand how to read these maps. Figure 4 illustrates an axial view example of the asymmetry maps direction definition. Let $X$ be an object (in green) and $S_{P}(X)$ its symmetrical image (in orange) according to the symmetry plane $P$. We compute the deformation superposing $X$ on $S_{P}(X)$ illustrated with the arrows in Figure 4. The left-right component ( $x$ axis) of the deformation field (arrows a, b, c, d) are all positive deformations because they all point towards the positive $x$ axis. The anterior-posterior component ( $y$ axis) of the deformation field has some positive values (arrows $\mathrm{f}, \mathrm{g}$ ) which point towards the positive $y$ axis and some negative values (arrows e, h) which point towards the negative $y$ axis.

The amplitudes of the deformation are illustrated using a color map (cold to hot type) as shown in Figure 4 color bar example. Asymmetry results use this color map over the brain mean shape of a population. This three components asymmetry maps definition is useful to highlight specific asymmetries in a chosen direction. However one needs to look carefully at the axis direction for asymmetry interpretation. Positive (resp. negative) values which lead to hot (resp. cold) colors do not necessary mean expansion (resp. shrinkage). Indeed, some deformations in Figure 4 (arrows e, f) fit with the intuitive interpretation of positive expansion and negative shrinkage, but other deformations (arrows $\mathrm{g}, \mathrm{h}$ ) are in the opposite direction.

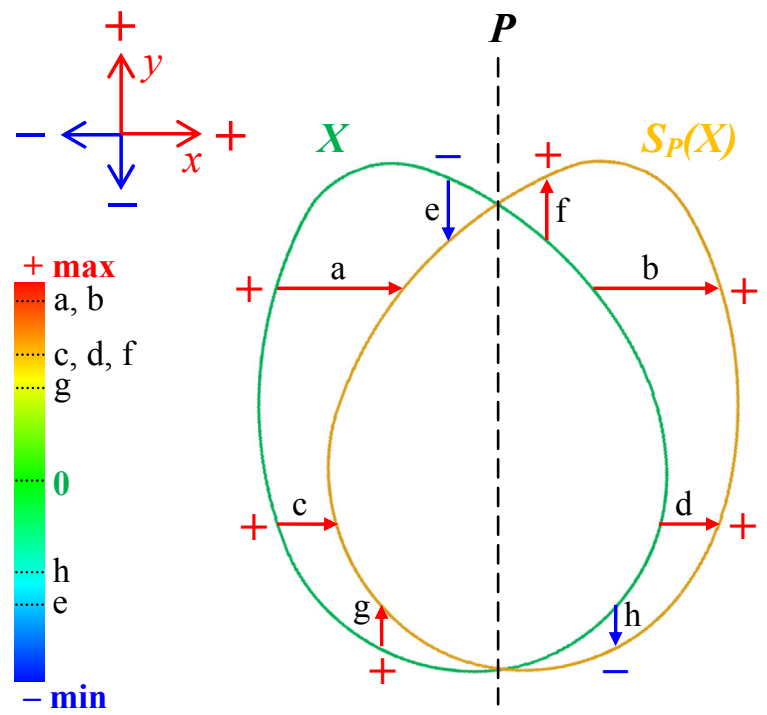

Fig. 4. Asymmetry maps direction definition. Orthogonal components of the deformation field which are in the same (resp. opposite) direction as their positive axis definition have positive (resp. negative) values. A color map is used to represent the signed scalar values of the deformation field.

Figure 5 illustrates the typical asymmetry color map of the Yakovlevian torque in the case of right-handed human subjects. The right-left and posterior-anterior components of the deformation field are shown according to the axes positive direction definitions shown in Figure 5. These two positive axes direction conventions are also the ones considered for all human and chimpanzee results presented. The convention for all results considered for the third component of the deformation field (not shown in Figure 5) is from Foot to Head. One can note that inverting the direction definition of a specific axis would result in inverting its respective red-blue asymmetry map pattern. 


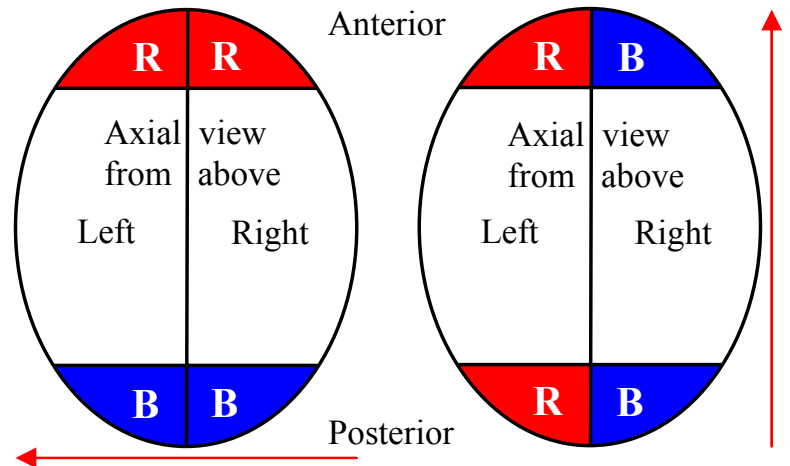

(a) Right to Left (b) Posterior to Anterior

Fig. 5. Asymmetry color map schematization. Typical Yakovlevian torque asymmetry map for right-handed human subjects. (a) Right-left component of the deformation field positively defined from Right to Left. (b)Posterior-anterior component of the deformation field positively defined from Posterior to Anterior.

\subsection{Humans and chimpanzees asymmetry results}

Figure 6 shows the most significant views (in the right-left and posterior-anterior components) of the asymmetry maps and p-values for human results. Figures 6(a) to 6(d) have indication of the positive direction of the deformation field. Their legend color bars indicate the signed deformation for each component and green color means no asymmetry (deformation). Each row shows one of the components and displays the region where the asymmetry is the most significant. The p-values maps are computed according to the t-test with the null hypothesis that there is no asymmetry at the significance level $\mathrm{p}=0.05$ (corrected). Red color indicates with more confidence regions with asymmetries. At the population level, the left occipital lobe appears to be wider and to have a posterior protrusion compared to the right occipital lobe. Asymmetry maps observations show a similar behavior of the right frontal lobe respectively to the left frontal lobe. However in frontal lobes the deformations are smaller and less equally distributed (Figures 6(a) and 6(c)) and the t-test (not shown) is not significant. These findings using our surfaced-based asymmetry method agree with the literature about the Yakovlevian torque.

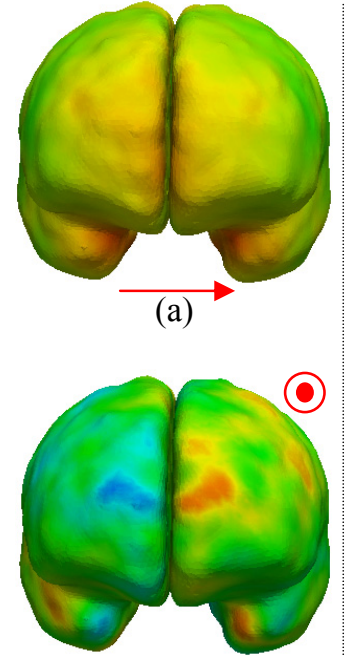

(c)

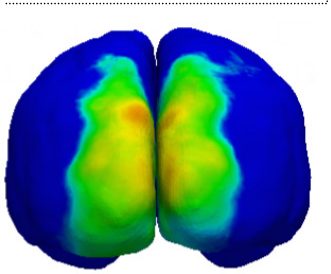

(e)

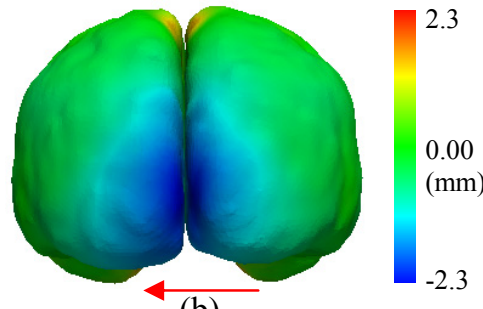

(b)

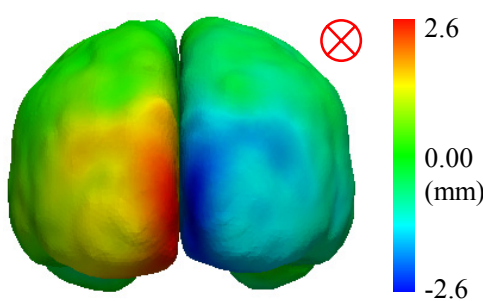

(d)

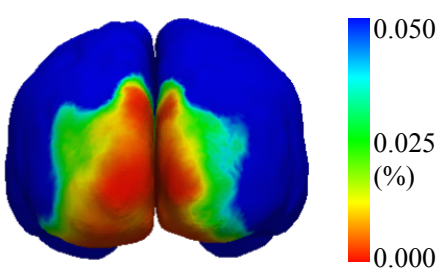

(f)
Fig. 6. Humans asymmetry maps and p-values. (a) Front view of right-left component. (b) Back view of right-left component. (c) Front view of posterior-anterior component. (d) Back view of posterior-anterior component. (e) p-values back view of right-left component (resp. to b). (f) p-values back view of posterior-anterior component (resp. to d).

Figure 7 shows equivalent results as in Figure 6 but with the chimpanzees. Very similar observations about the width and protrusion patterns of the frontal and occipital lobes in humans can be seen throughout the chimpanzee results. However, two differences are observed mainly in the frontal lobes (Figures 7(a) and 7(c)) where the greater values of deformations are not located at the tip of the lobes but moved laterally on each side. Moreover this time the most significant t-test results were observed in both frontal and occipital lobes of the right-left component (Figures 7(e) and 7(f)) compared to the test on the posterior-anterior component (not shown). An interesting observation is that the maximum deformation amplitude for each component is almost the same in chimpanzees and humans even if the human brain size and volume is greater. In that sense, this suggests the chimpanzee brain would be more asymmetrical than the human one. Nevertheless, according to our surfaced-based asymmetry method findings, it seems that the Yakovlevian torque pattern of the human brain is also present in the chimpanzee brain, at least in our study on this adult male right-handed subgroup. 


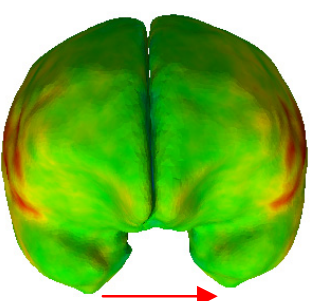

(a)

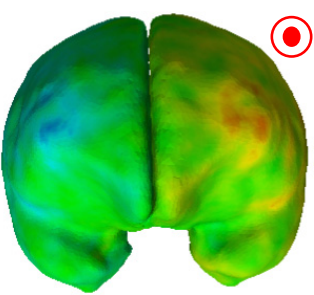

(c)

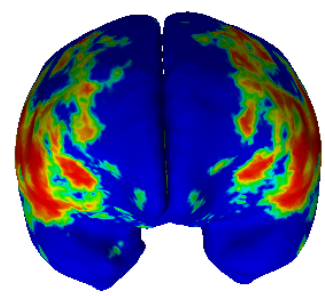

(e)

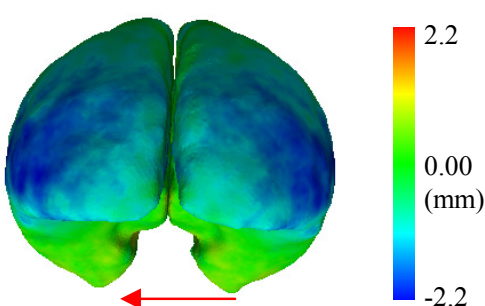

(b)

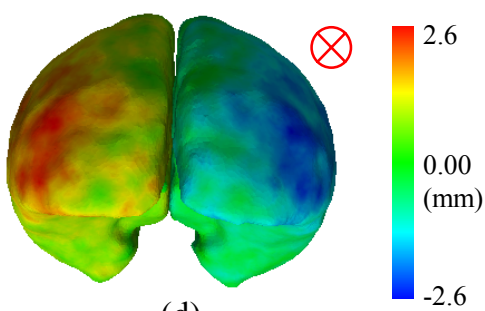

(d)

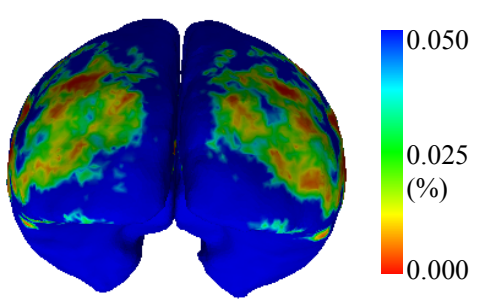

(f)
Fig. 7. Chimpanzees asymmetry maps and p-values.

(a) Front view of right-left component. (b) Back view of right-left component. (c) Front view of posterior-anterior component. (d) Back view of posterior-anterior component. (e) p-values front view of right-left component (resp. to a). (f) p-values back view of right-left component (resp. to b).

Figure 8 shows an interesting finding using our surfacebased asymmetry method on humans in the occipital lobe area with a significant foot-head (vertical) asymmetry. The left lobe seems to be greater in the lower vertical direction compared to its counterpart. This observation can be seen as an addition to the Yakovlevian torque where the left occipital lobe is already greater in the sense that it is wider and that it protrudes more in the posterior direction compared to the right lobe. This observation is not present at all in the chimpanzees and compared to the previous results, it is the first significant difference finding between the human and chimpanzee brains using our surfaced-based asymmetry method. We also notice that the maximum asymmetry $(1.7 \mathrm{~mm})$ is smaller than the other components of the deformation field in humans. The equivalent maximum foot-head deformation in chimpanzees (1.3mm, not shown) is also significantly smaller than the human one, compared to the other components where maximum values were almost identical in both populations.

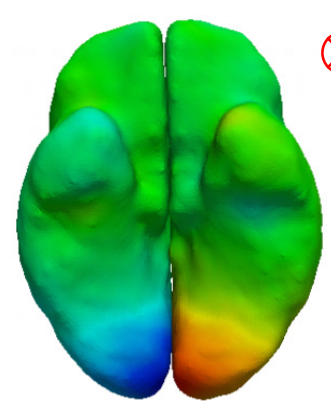

(a)

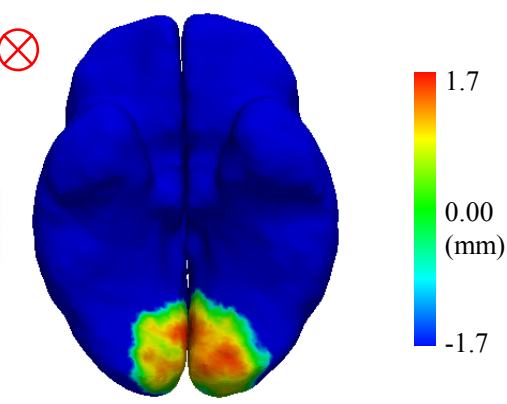

(b)
Fig. 8. Humans foot-head deformation field component. (a) Bottom view of asymmetry map. (b) Bottom view of pvalues. The color bar legend is for the asymmetry map. Pvalues have the same legend as previous figures $(0 ; 0.05)$.

Figure 9 shows how significant are the deformation fields in human and chimpanzee on the left hemisphere for the right-left component. It is interesting to see that our surface-based method results show very similar and significant specific asymmetry in the right-left component of the deformation field around Broca's area in both populations. However the amplitude of the deformation in the right-left component is small in that area, especially in humans where it is almost not visible on the asymmetry map when displayed at the regular full asymmetry range. The specific asymmetry observation in Figure 9 is only present in that component of the deformation field. For both populations there is no significant asymmetry in the other two components around Broca's area.

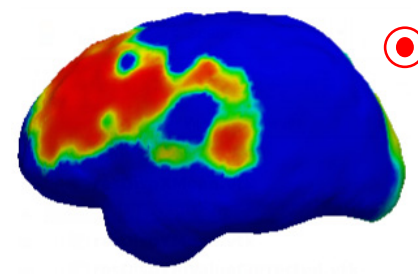

(a)

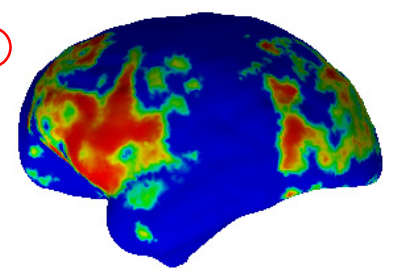

(b)
Fig. 9. Chimpanzees and humans right-left p-values.

(a) Humans left side view. (b) Chimpanzees left side view. $\mathrm{P}$-values have the same legend as previous figures $(0 ; 0.05)$ for both populations.

\section{CONCLUSION}

In this paper we proposed a new surface-based method to compute bilateral brain asymmetries with respect to the midsagittal plane. We used this method on both chimpanzee and human populations in order to compare their asymmetries. We adapted a human brain segmentation pipeline to work with chimpanzee MR images. We proposed a brain global shape mesh processing algorithm to compute a brain hull in order to alleviate the problems due to cortical variability. 
We designed a method for mid-sagittal plane computation and individual brain asymmetry evaluation and a method for the estimation of a brain mean shape used to map statistical asymmetries at the population level. Results are presented as specific asymmetry maps for each component of the deformation field between the brain and its mirrored image. Our method allowed recovering typical global human brain asymmetry patterns previously observed and to find another specific asymmetry component. Future work will involve more extensive studies on a larger database including comparison with other subgroups of both investigated populations and also with other great apes populations. We will design an automated comparison method between populations including an integration of asymmetry on specific anatomical regions for easier interpretation of the results.

\section{REFERENCES}

[1] Geschwind, N., Levitsky, W.: Human brain: left-right asymmetries in temporal speech region. Science 161(837) (July 1968) 186-167

[2] LeMay, M.: Functional and anatomical asymmetries of the human brain. Eur J Neurol 6(1) (January 1999) 79-85

[3] Toga, A., Thompson, P.: Mapping brain asymmetry. Nature Reviews Neuroscience 4(1) (January 2003) 37-48

[4] Holloway, R.L., Costelareymondie, M.C.D.L.: Brain endocast asymmetry in pongids and hominids: some preliminary findings on the paleontology of cerebral dominance. Am J Phys Anthropol 58(1) (May 1982) 101-10

[5] Crow, T.: Directional asymmetry is the key to the origin of modern Homo sapiens (the Broca-Annett axiom): A reply to Rogers' review of The Speciation of Modern Homo Sapiens. Laterality 9(2) (April 2004) 233-242

[6] Gannon, P.J., Holloway, R.L., Broadfield, D.C., Braun, A.R.: Asymmetry of chimpanzee planum temporale: humanlike pattern of Wernicke's brain language area homolog. Science 279(5348) (January 1998) 220-222

[7] Cantalupo, C., Hopkins, W.D.: Asymmetric Broca's area in great apes. Nature 414(6863) (November 2001) 505

[8] Good, C., Johnsrude, I., Ashburner, J., Henson, R., Friston, K., Frackowiak, R.: Cerebral asymmetry and the effects of sex and handedness on brain structure: a voxel-based morphometric analysis of 465 normal adult human brains. NeuroImage 14(3) (September 2001) 685-700

[9] Watkins, K., Paus, T., Lerch, J., Zijdenbos, A., Collins, D., Neelin, P., Taylor, J., Worsley, K., Evans, A.: Structural asymmetries in the human brain: a voxel-based statistical analysis of 142 MRI scans. Cereb Cortex 11(9) (September 2001) 868-877

[10] Herve, P.Y., Crivello, F., Perchey, G., Mazoyer, B., TzourioMazoyer, N.: Handedness and cerebral anatomical asymmetries in young adult males. Neuroimage 29(4) (February 2006) 1066-1079

[11] Thirion, J.P., Prima, S., Subsol, G., Roberts, N.: Statistical analysis of normal and abnormal dissymmetry in volumetric medical images. Medic Image Analysis 4(2) (June 2000) 111-121
[12] Prima, S., Ourselin, S., Ayache, N.: Computation of the midsagittal plane in 3D brain images. IEEE Transactions on Medical Imaging 21(2) (February 2002) 122-138

[13] Barrick, T.R., Mackay, C.E., Prima, S., Maes, F., Vandermeulen, D., Crow, T.J., Roberts, N.: Automatic analysis of cerebral asymmetry: an exploratory study of the relationship between brain torque and planum temporale asymmetry. Neuroimage 24(3) (February 2005) 678-691

[14] Hopkins, W.D., Taglialatela, J.P., Meguerditchian, A., Nir, T., Schenker, N.M., Sherwood, C.C.: Gray matter asymmetries in chimpanzees as revealed by voxel-based morphometry. Neuroimage 42(2) (August 2008) 491-497

[15] Avants, B.B., Schoenemann, P.T., Gee, J.C.: Lagrangian frame diffeomorphic image registration: Morphometric comparison of human and chimpanzee cortex. Med Image Anal 10(3) (June 2006) 397-412

[16] Keller, S.S., Roberts, N., Hopkins,W.: A comparative magnetic resonance imaging study of the anatomy, variability, and asymmetry of broca's area in the human and chimpanzee brain. J Neurosci 29(46) (November 2009) 14607-14616

[17] Hopkins, W.D., Lyn, H., Cantalupo, C.: Volumetric and lateralized differences in selected brain regions of chimpanzees (pan troglodytes) and bonobos (pan paniscus). Am J Primatol 71(12) (December 2009) 988-997

[18] Schenker, N.M., Hopkins, W.D., Spocter, M.A., Garrison, A.R., Stimpson, C.D., Erwin, J.M., Hof, P.R., Sherwood, C.C.: Broca's area homologue in chimpanzees (pan troglodytes): probabilistic mapping, asymmetry, and comparison to humans. Cereb Cortex 20(3) (March 2010) 730-742

[19] Granger, S., Pennec, X.: Multi-scale EM-ICP: A fast and robust approach for surface registration. Lecture Notes in Computer Science 2353(1) (May 2002) 418-432

[20] Combès, B., Hennessy, R., Waddington, J., Roberts, N., Prima, S.: Automatic symmetry plane estimation of bilateral objects in point clouds. IEEE Conference on Computer Vision and Pattern Recognition, Anchorage, USA (June 2008) 1-8

[21] Combès, B., Prima, S.: Setting priors and enforcing constraints on matches for nonlinear registration of meshes. 12th International Conference on Medical Image Computing and Computer-Assisted Intervention, London, UK (September 2009) $175-183$

[22] Combès, B., Fournier, M., Kennedy, D.N., Braga, J., Roberts, N. and Prima, S. : EM-ICP strategies for joint mean shape and correspondences estimation: applications to statistical analysis of shape and of asymmetry. ISBI'2011: 8th IEEE International Symposium on Biomedical Imaging, Chicago, USA (April 2011), to appear.

[23] Nichols, T., Holmes, A.: Nonparametric permutation tests for functional neuroimaging: A primer with examples. Human Brain Mapping 15(1) (July 2001) 1-25 